\title{
Marked and prolonged serotonin toxicity in a tramadol-poisoned patient with a pharmacokinetic study
}

Guillaume Bianconi ${ }^{\mathrm{a}}$, Isabelle Malissin ${ }^{\mathrm{b}, \mathrm{c}}$, Laurence Labat ${ }^{\mathrm{c}, \mathrm{d}}$, Nihel Khoudour ${ }^{\mathrm{a}}$, Pascal Houzéd, Nicolas Pallet $^{\mathrm{e}, \mathrm{f}}$, Bruno Mégarbane $\mathrm{b}^{\mathrm{b}, \mathrm{c}, *}$, Xavier Declèves ${ }^{\mathrm{a}, \mathrm{c}, *}$

aBiologie du Médicament, AP-HP, Hôpital Cochin, Paris, France, ${ }^{b}$ Réanimation Médicale et Toxicologique, Hôpital Lariboisière, Fédération de Toxicologie de l'AP-HP, Paris, France, 'Université de Paris, Inserm, UMRS-1144, Paris, France, 'Laboratoire de Toxicologie, AP-HP, Hôpital Lariboisière, Paris, France, eService de Biochimie, AP-HP, Hôpital Européen Georges Pompidou, F-75015, Paris, France, fUniversité de Paris, INSERM U1138, Centre de Recherche des Cordeliers, Paris, France.

*These two authors equally contributed to the manuscript.

CONTACT: Prof. Bruno Mégarbane. Réanimation Médicale et Toxicologique, Hôpital Lariboisière, 2 Rue Ambroise Paré, 75010 Paris, France. Telephone: +33-149958961; Fax: +33-149956578. E-mail: bruno.megarbane@lrb.aphp.fr

\section{Funding: None}

Authors' contributions: B.M. and X.D. designed the study. I.M. and B.M. managed the patient. G.B., L.L. N.K., P.H., and X.D. performed the toxicological analyses. N.P. performed the genotyping. G.B. and X.D. performed the pharmacokinetic study. G.B. drafted the manuscript. B.M. and X.D. revised the initial version. All authors contributed to writing the manuscript and agreed with its final version.

Disclosure statement: The authors report no conflict of interest. The authors alone are responsible for the content and writing of this paper. The manuscript has been read and approved by all authors. The authors certify that the submission is not under review at any other publication. The authors certify that the authors have no other submissions and previous reports that might be regarded as overlapping with the current work. The authors declare no financial disclosures.

Running head: Tramadol-related serotonin toxicity

Word count: 1,204 words, 12 references, 2 figures + supplemental data 


\begin{abstract}
BACKGROUND Tramadol poisoning rarely causes serotonin toxicity, which mechanisms remain poorly understood. We investigated alterations in tramadol pharmacokinetics in a tramadol-poisoned patient who presented with marked and prolonged serotonin toxicity.

CASE REPORT A 21-year-old male self-ingested 750mg-tramadol, 200mg-sotalol, 400mg-propranolol and 6mg-lorazepam. He was a kidney transplant patient treated with mycophenolate, tacrolimus, prednisone and paroxetine. He developed transitory cardiovascular failure and prolonged serotonin toxicity requiring sedation, muscle paralysis and cyproheptadine, with favorable outcome.
\end{abstract}

METHODS We measured plasma concentrations of tramadol, M1, M2 and M5 using liquid-chromatographytandem mass spectrometry, calculated elimination half-lives and metabolic ratios of the compounds, and genotyped cytochromes involved in tramadol metabolism.

RESULTS Elimination half-lives of tramadol (6.1h) and M1 (7.1h) were normal while those of M2 (26.5h) and M5 (16.7h) prolonged. M1 metabolic ratio (0.12) was 2-fold reduced, M2 metabolic ratio (197) 1,000fold increased and M5 metabolic ratio (0.12) normal. This metabolic profile in a patient with normal CYP2D6-metabolizer status based on genotyping supports CYP2D6 inhibition by paroxetine and propranolol, two strong mechanism-based inhibitors. Only M2 present in sufficient concentrations up to $48 \mathrm{~h}$ could explain the prolonged serotonin toxicity.

CONCLUSION Marked and prolonged serotonin toxicity was attributed to increased M2 production due to paroxetine- and propranolol-related CYP2D6 inhibition of tramadol metabolism.

KEYWORDS Tramadol; Serotonin syndrome; Poisoning; Drug-drug interaction; Pharmacokinetics 
Tramadol is a commonly prescribed centrally acting opioid with safe profile. Mechanisms of tramadolrelated analgesia and toxicity are likely related to its ability to block serotonin and norepinephrine reuptake and to the elevated affinity of its $O$-desmethylated metabolite (M1) to mu-opioid receptors [1]. Tramadol poisoning is mainly responsible for massive opioid-like central nervous system (CNS) depression and seizures, whereas serotonin toxicity is rare and with poorly understood reasons [2,3].

Tramadol is rapidly absorbed (peak, 1-2h; bioavailability, 70\%) with linear pharmacokinetics in the 50400mg dose range [1]. Tramadol undergoes extensive liver oxidative metabolism, which produces both M1 by cytochrome 2D6 (CYP2D6) and $N$-desmethyltramadol (M2) by CYP3A4 and CYP2B6. Subsequently, $\mathrm{M} 1$ and $\mathrm{M} 2$ are metabolised into $\mathrm{N}, \mathrm{N}$-didemethyltramadol (M3) and $\mathrm{N}, \mathrm{O}$-didemethyltramadol (M5), respectively, then into $\mathrm{N}, \mathrm{N}, \mathrm{O}$-tridesmethyltramadol (M4) (Fig.1). M1 is inactivated by glucuronidation, mainly by two UDP-glucuronosyltransferases UGT2B7 and UGT1A8. Elimination half-lives of tramadol and $\mathrm{M} 1$ are close (6-8h) since M1 is a formation-dependent tramadol metabolite.

CYP2D6 plays a key-role in tramadol pharmacokinetics and analgesic efficacy as it produces M1. In overdose, CYP2D6 activity is saturated due to its small contribution to total liver CYP activity ( 1-5\%). Poor CYP2D6-metabolisers have less analgesia with tramadol compared to normal CYP2D6 activity individuals, in relation to deleterious CYP2D6 allelic variants, which result in increased exposure to tramadol and reduced M1 formation [1]. By contrast, ultra-rapid CYP2D6 metabolizers are at increased risk opioid toxicity. Moreover, drugs acting as inhibitors or inducers of CYP2D6, CYP3A4 and CYP2B6 are at risk of deleterious drug-drug interactions (DDIs). Toxicity has been associated with higher plasma M1 metabolic ratio, which increases mu-opioid receptor occupancy [4].

We report a tramadol-poisoned patient who developed marked and prolonged serotonin toxicity in relation to DDI that dramatically altered tramadol pharmacokinetics.

\section{Case Report}

A 21-year-old patient was admitted to our intensive care unit two hours after ingesting $750 \mathrm{mg}$ tramadol, $200 \mathrm{mg}$ sotalol, $400 \mathrm{mg}$ propranolol and $6 \mathrm{mg}$ lorazepam in a suicidal attempt. He was a kidney transplant patient treated daily with $250 \mathrm{mg}$ mycophenolate mofetil, $4 \mathrm{mg}$ tacrolimus, $5 \mathrm{mg}$ prednisone, $20 \mathrm{mg}$ paroxetine, and 3mg lorazepam. On admission, he was sleepy (Glasgow coma score, 13), slightly bradycardic $(50 \mathrm{bpm})$ and hypotensive $(86 / 43 \mathrm{mmHg})$. His body temperature was $36.7^{\circ} \mathrm{C}$. Physical examination showed hypertonia, tremor, mydriasis, spontaneous and inducible myoclonus, diffuse hyperreflexia, patellar clonus, and bilateral Babinski sign, all suggestive of marked serotonin toxicity using either Sternbach's criteria [5] or Hunter serotonin toxicity criteria [6]. Laboratory tests showed serum creatinine of $109 \mu \mathrm{mol} / \mathrm{L}$ (clearance, $83 \mathrm{~mL} / \mathrm{min}$ ), blood lactate of $0.8 \mathrm{mmol} / \mathrm{L}$ and normal liver and coagulation tests. Drug screening was negative except for tramadol with estimated concentration of $400 \mu \mathrm{g} / \mathrm{L}$ 
(therapeutic range, $100-800 \mu \mathrm{g} / \mathrm{L}$; toxic range, $>1,000 \mu \mathrm{g} / \mathrm{L}$ ). Plasma paroxetine was $58 \mu \mathrm{g} / \mathrm{L}$ (therapeutic range, 2-60; liquid chromatography-mass spectrometry high-resolution). Using immunoassays, plasma tacrolimus and mycophenolate were $10.5 \mu \mathrm{g} / \mathrm{L}$ (therapeutic range, 5.0-15.0) and 1,000 $\mathrm{g} / \mathrm{L}$ (therapeutic range, 1,000-3,000), respectively. Using high-liquid chromatography-fluorescence detection, plasma sotalol and propranolol were $13,500 \mu \mathrm{g} / \mathrm{L}$ (therapeutic range, 1,000-2,000) and $738 \mu \mathrm{g} / \mathrm{L}$ (therapeutic range, 45 170), respectively.

Rapidly, the patient developed cardiovascular failure requiring mechanical ventilation, isoprenaline (peak infusion rate, $20 \mu \mathrm{g} / \mathrm{kg} / \mathrm{min}$ ) and norepinephrine (peak infusion rate, $1 \mu \mathrm{g} / \mathrm{kg} / \mathrm{min}$ ) infusions guided by echocardiography. Electroencephalography evidenced no seizure activity. Despite deep sedation, serotonin toxicity including myoclonus persisted requiring muscle paralysis and cyproheptadine administration (12mg followed by $4 \mathrm{mg}$ t.i.d by gastric tube). Blood pressure and cardiac function improved allowing catecholamine weaning on day2. Renal and liver functions remained unaltered. Serotonin toxicity reversed progressively allowing cyproheptadine weaning on day4, extubation on day6 and discharge to the psychiatric ward on day7. On 3-month follow-up, the patient had fully recovered and returned to his original activities.

\section{Methods}

Plasma concentrations of tramadol, M1, M2 and M5 were measured from 17.5 to $105.5 \mathrm{~h}$ after the reported ingestion time (Fig.2), using liquid-chromatography-tandem mass spectrometry (QuantumUltra ${ }^{\mathrm{TM}}$ mass spectrometer equipped with an ion maxelectrospray interface, ThermoFisher Scientific, Waltham, Ma) [7]. Plasma elimination half-lives and areas under the curve (AUCs, from the first to the last sampling time, extrapolated to infinity) were estimated using non-compartmental pharmacokinetic analyses (Phoenix WinNonlin ${ }^{\mathrm{TM}}$, Certera, St Louis, MO). The ratio of plasma metabolite-to-tramadol concentration AUCs was determined as measure of the drug-metabolizing enzymes that form and clear each compound. Methods of CYP genotyping based on allelic discrimination are explained in the supplementary material.

\section{Results and discussion}

Elimination half-lives of tramadol $(6.1 \mathrm{~h})$ and M1 (7.1h) were similar to published values [1], whereas elimination half-lives of M2 (26.5h) and M5 (16.7h) were prolonged in comparison to published values ( 910h) [8], suggesting reduced clearance in our tramadol-poisoned patient. M1 metabolic ratio (0.12) was more than 2 times lower than those reported $(\sim 0.27)$ [8], but close to that reported when tramadol is comedicated with strong CYP2D6 inhibitors ( 0.14) [8]. By contrast, M2 metabolic ratio was markedly elevated (197), i.e. at least 1,000 times higher than those reported ( 0.13) [8], even though increase in this 
ratio could also be related to high-dose tramadol exposure, as shown in a rat model [7]. Finally, M5 metabolic ratio was 0.12 , similar to published values ( 0.12) [8].

Genotyping revealed that the patient was normal/rapid CYP2B6 and CYP2D6 metabolizer, homozygous CYP3A4 wild-type and homozygous for mutations associated with non-expressing intestinal CYP3A5, altogether supporting that tramadol elimination could not be slowed down by gene alteration. Precisely, CYP2D6 genotype was $* 2 / * 41$, a diplotype associated with an activity score of 1.5 , predictive of normal function according to the Clinical Pharmacogenetics Implementation Consortium guideline for CYP2D6 (https://cpicpgx.org/guidelines/guideline-for-codeine-and-cyp2d6/).

In our patient chronically treated with paroxetine, a potent selective serotonin reuptake inhibitor, tramadol overdose resulted in marked serotonin toxicity, probably caused by additional serotonin accumulation in the synaptic cleft. Interestingly, low M1 metabolic ratio, not explained by poor metabolic CYP2D6 activity, could be attributed to CYP2D6 activity inhibition that may have blocked CYP2D6-mediated M1 formation and subsequently facilitated CYP3A4- and/or CYP2B6-mediated tramadol metabolism into M2. Paroxetine and propranolol, two strong selective mechanism-based CYP2D6 inhibitors [9,10], could have contributed to such alterations.

Tramadol elimination half-life was consistent with published data [1], supporting its adequate elimination by all metabolic pathways. Inhibition of CYP2D6-mediated metabolism of M2 to M5 explained prolonged M2 elimination half-life together with the huge increase in its metabolic ratio. Subsequently, M5 elimination half-life was increased since its formation is depending on M1 and M2 production. Interestingly, considering estimated half-lives and plasma exposures to tramadol and metabolites, only M2 could be present in sufficient concentrations up to $48 \mathrm{~h}$ to explain the prolonged serotonin toxicity, which lasted up to $48 \mathrm{~h}$ postingestion, especially since plasma tramadol was $<10 \mathrm{ng} / \mathrm{mL}$ and $\mathrm{M} 1$ not detectable at that time. Although the exact toxic effects of M2 are poorly understood, our data provide evidence to support its possible contribution to tramadol-attributed serotonin toxicity in addition to the parent compound.

Other co-medicated drugs did not likely contribute to the alterations in tramadol pharmacokinetics. Importantly, tacrolimus had no effect on CYPs at concentrations $<1 \mu \mathrm{mol} / \mathrm{L}(804 \mathrm{ng} / \mathrm{mL})$, while at higher concentrations, it may exhibit mild CYP3A4- and CYP3A5-related inhibitory effects [11]. Similarly, no DDI involving mycophenolate with CYP-mediated tramadol metabolism but UGT induction in tuberculosis patients treated with rifampicin have been described [12].

To conclude, our findings suggest that severity and prolonged duration of serotonin toxicity in our tramadol-poisoned patient can be attributed to co-medicated paroxetine and co-ingested propranolol-related CYP2D6 inhibition that resulted in the reduction of CYP2D6-produced M1 and consequent increase in CYP3A4- and CYP2B6-produced but CYP2D6-eliminated M2. Cumulative serotoninergic effects between drugs could have additionally contributed to the overall serotonin toxicity. 


\section{References}

1- Doostmohammadi M, Rahimi HR. ADME and toxicity considerations for tramadol: from basic research to clinical implications. Expert Opin Drug Metab Toxicol. 2020;16:627-640.

2- Ryan NM, Isbister GK. Tramadol overdose causes seizures and respiratory depression but serotonin toxicity appears unlikely. Clin Toxicol (Phila). 2015;53:545-50.

3- Mégarbane B, Lagard C, Chevillard L. Tramadol: Distinguishing the Pathophysiology of Serotonin Syndrome and Seizures. Am J Med. 2018;131:e487.

4- Ahmadimanesh M, Naeini MB, Rouini MR, Shadnia S, Ghazi-Khansari M. Assessment of tramadol pharmacokinetics in correlation with CYP2D6 and clinical symptoms. Drug Metab Pers Ther. 2020;35:/j/dmdi.2020.35.issue-2/dmpt-2019-0021/dmpt-2019-0021.xml.

5- Sternbach H. The serotonin syndrome. Am J Psychiatry. 1991;148:705-13.

6- Dunkley EJ, Isbister GK, Sibbritt D, Dawson AH, Whyte IM. The Hunter Serotonin Toxicity Criteria: simple and accurate diagnostic decision rules for serotonin toxicity. QJM. 2003;96:635-42.

7- Lagard C, Chevillard L, Malissin I, Risède P, Callebert J, Labat L, Launay JM, Laplanche JL, Mégarbane B. Mechanisms of tramadol-related neurotoxicity in the rat: Does diazepam/tramadol combination play a worsening role in overdose? Toxicol Appl Pharmacol. 2016;310:108-119.

8- Hagelberg NM, Saarikoski T, Saari TI, Neuvonen M, Neuvonen PJ, Turpeinen M, Scheinin M, Laine K, Olkkola KT. Ticlopidine inhibits both O-demethylation and renal clearance of tramadol, increasing the exposure to it, but itraconazole has no marked effect on the ticlopidine-tramadol interaction. Eur $\mathbf{J}$ Clin Pharmacol. 2013;69:867-75.

9- Tolledo EC, Miksys S, Gonzalez FJ, Tyndale RF. Propranolol is a mechanism-based inhibitor of CYP2D and CYP2D6 in humanized CYP2D6-transgenic mice: Effects on activity and drug responses. Br J Pharmacol. 2020;177:701-712.

10- Bertelsen KM, Venkatakrishnan K, Von Moltke LL, Obach RS, Greenblatt DJ. Apparent mechanismbased inhibition of human CYP2D6 in vitro by paroxetine: comparison with fluoxetine and quinidine. Drug Metab Dispos. 2003;31:289-93.

11- Degraeve AL, Moudio S, Haufroid V, Chaib Eddour D, Mourad M, Bindels LB, Elens L. Predictors of tacrolimus pharmacokinetic variability: current evidences and future perspectives. Expert Opin Drug Metab Toxicol. 2020;16:769-782.

12- Kuypers DR, Verleden G, Naesens M, Vanrenterghem Y. Drug interaction between mycophenolate mofetil and rifampin: possible induction of uridine diphosphate-glucuronosyltransferase. Clin Pharmacol Ther. 2005;78:81-8. 


\section{Figure legend}

Fig.1. Drug-metabolizing enzymes involved in the metabolic pathway of tramadol.

M1, O-desmethyltramadol; $\mathrm{M} 2, \mathrm{~N}$-desmethyltramadol; $\mathrm{M} 3, \mathrm{~N}, \mathrm{~N}$-didemethyltramadol; $\mathrm{M} 4 ; \mathrm{N}, \mathrm{N}, \mathrm{O}$ tridesmethyltramadol; $\mathrm{M} 5, \quad \mathrm{~N}, \mathrm{O}$-didemethyltramadol; $\mathrm{CYP}$, cytochrome P450; UGT, UDPglucuronosyltransferase; ?, the exact involved enzyme is uncertain

Fig.2. Pharmacokinetics of tramadol and its three metabolites M1, M2 and M5 in plasma in a tramadolpoisoned patient with marked and prolonged serotonin toxicity 


\section{Supplementary material:}

\section{Methods used for cytochrome P450 genotyping:}

For genetic analyses, DNA was extracted from peripheral blood leukocytes using the Blood DNA kit ${ }^{\mathrm{TM}}$ (Qiagen, Courtaboeuf, France) in accordance with standard protocols. Genotyping of the CYP3A5*3, CYP3A4*22 and CYP2B6*5 and *6 alleles was performed using TaqMan Assay Reagents ${ }^{\mathrm{TM}}$ for allelic discrimination (Applied Biosystems, Courtaboeuf, France) with a 7900HT Applied Biosystems thermal cycler. CYP2D6 molecular alterations were characterized using targeted Next Generation Sequencing (Ion AmpliSeq ${ }^{\mathrm{TM}}$ Custom IAD68279, Life Technologies, Carlsbad, CA). Briefly, the multiplex barcoded libraries were generated from 10ng DNA according to the manufacturer's recommendations (Ion ampliseq library kit V2) and normalized using the Ion Library Equalizer ${ }^{\mathrm{TM}}$ Kit. The pooled libraries (max 96) were processed with the Ion Chef ${ }^{\mathrm{TM}}$ System for template preparation and chip loading (Ion PI HI-Q Chef Kit, Ion PI Chip V3) and sequenced using the Ion Proton ${ }^{\mathrm{TM}}$ System (Life Technologies, Carlsbad, CA). A custom Ion Torrent panel has been designed with the Ampliseq Designer software using human hg19 as reference. The FASTQ sequencing data were aligned to the human genome (hg19) and processed using Ion Torrent Suite V5.0.4.0. CYP2D6 copy number variations (duplication and deletion) were evaluated with the Applied Biosystems TaqMan Copy Number Assays that use TaqMan MGB probe chemistry; TaqMan Copy Number Assays being run together with a TaqMan Copy Number Reference Assay in a duplex qPCR reaction. 


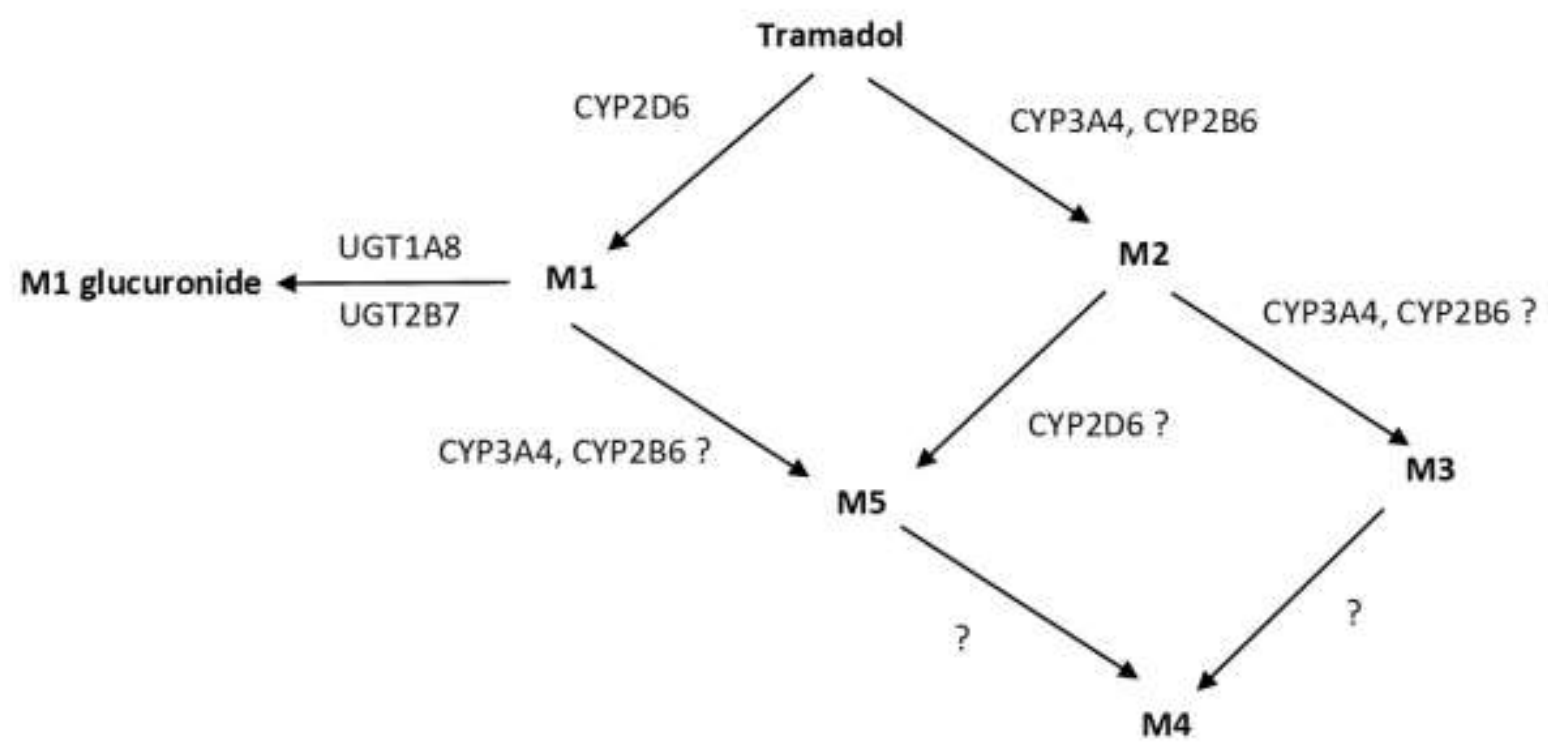

Figure 1

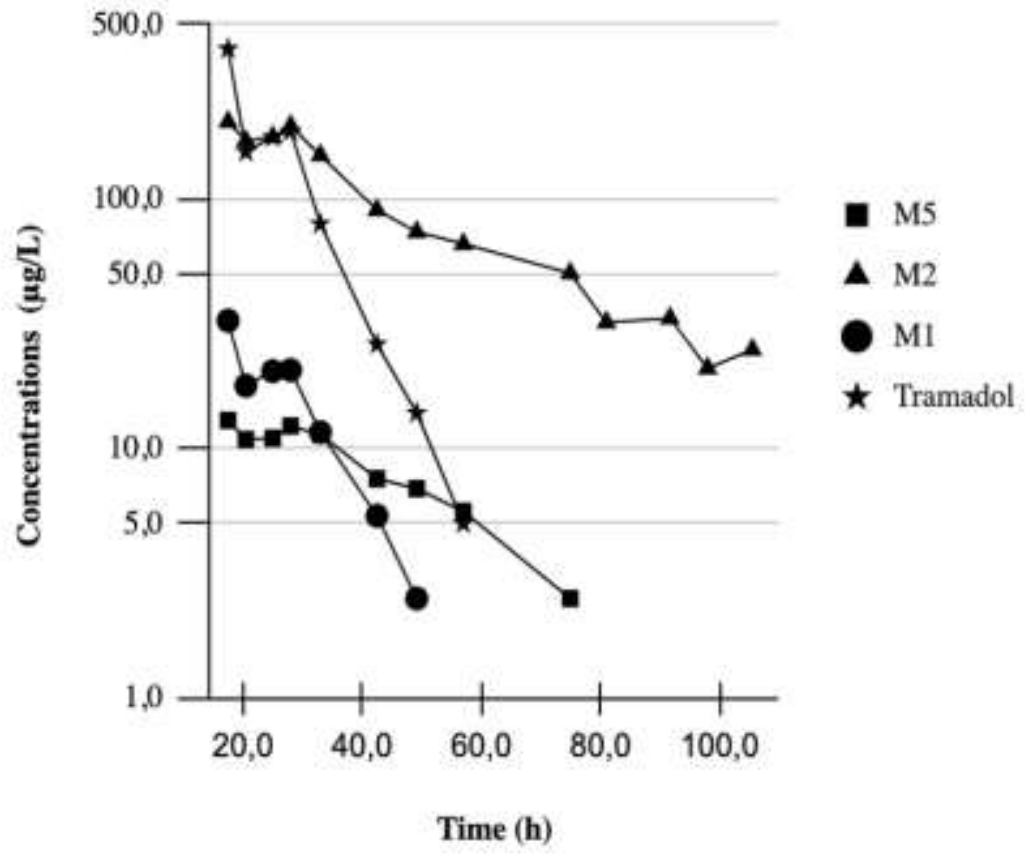

Fig 2 